\title{
Influence of absorption in linear polarization imaging of melanoma tissues
}

\author{
Dongzhi Li*,†, Honghui He*, Nan Zeng*, Weidong Xie*, \\ Ran Liao*, Jian $\mathrm{Wu}^{*}$, Yonghong $\mathrm{He}^{*}$ and Hui $\mathrm{Ma}^{*, \dagger}$, \\ *Shenzhen Key Laboratory for Minimal Invasive Medical Technologies \\ Graduate School at Shenzhen, Tsinghua University \\ Shenzhen 518055, P. R. China \\ ${ }^{\dagger}$ Department of Physics, Tsinghua University \\ Beijing 100084, P. R. China \\ ¥mahui@tsinghua.edu.cn
}

Received 20 October 2013

Accepted 4 November 2013

Published 16 December 2013

\begin{abstract}
The contrast mechanism of different polarization imaging techniques for melanoma in mouse skin is studied using both experiments and Monte Carlo simulations. Total intensity, linear polarization difference imaging (DPI), degree of polarization imaging (DOPI) and rotating linear polarization imaging (RLPI) are applied and the relative contrasts of these polarization imaging methods between the normal and cancerous tissues are compared. A two-layer absorption-scattering model is proposed to explain the contrast mechanism of the polarization imaging for melanoma. By taking into account of both scattering of symmetrical and asymmetrical scatterers and absorption of inter-scatterer medium, the two-layer model reproduces the relative contrasts for polarization images observed in experiments. The simulation results also show that, the parameters of polarization imaging change more dramatically with the variation of absorption in the bottom layer than the top layer.
\end{abstract}

Keywords: Polarization imaging; melanoma; two-layer model; Monte Carlo simulation.

\section{Introduction}

Optical properties of biological tissues are often affected by their physiological and pathological statuses. $^{1-4}$ Thus the normal and diseased tissues can be distinguished by measuring the changes of physical parameters in the interaction between light and biological tissues. ${ }^{5,6}$ However, as most of the biological tissues are highly turbid in the visible and near-infrared region, multiple scattering of photons will degrade the contrasts of optical images. ${ }^{7}$ The

This is an Open Access article published by World Scientific Publishing Company. It is distributed under the terms of the Creative Commons Attribution 3.0 (CC-BY) License. Further distribution of this work is permitted, provided the original work is properly cited. 
polarization imaging methods can suppress contributions from multiple scattered photons, thus improve the imaging quality for superficial tissues. ${ }^{7,8}$

The polarization imaging methods mainly obtain the information from the superficial layer of tissues, where epithelial lesions start to develop. ${ }^{9}$ The rich information of different polarization imaging methods offers them good potential in clinical diagnosis of early lesions. ${ }^{7-12}$ For example, Anderson ${ }^{10}$ developed the difference polarization imaging method (DPI), in which the difference of two images with perpendicular polarization states is calculated. Jacques et al. ${ }^{7}$ proposed the degree of polarization imaging method (DOPI), in which the difference of polarization is normalized. Both DPI and DOPI methods have been applied in demarcating the margins of cancerous tissues. ${ }^{7-10}$ Recently, we have developed rotating linear polarization imaging (RLPI) and applied it to cancerous liver tissues. ${ }^{11,12}$ The RLPI method provides a new set of parameters for quantitative characterization of the optical and structural properties for biological tissues and a new tool for clinical biomedical diagnosis. ${ }^{11-13}$

Skin cancer often occurs in superficial epithelial tissue, therefore is suitable for polarization imaging methods such as DPI and DOPI. ${ }^{8,10}$ Melanoma is one of the most common skin cancers. The strong absorption of melanin in melanoma has significant impact on imaging. To analyze the imaging data of tissues with strong absorption, two-layer skin scattering models have been proposed. ${ }^{14-18}$ Zonios et al. ${ }^{15,16}$ presented a nonpolarized isotropic skin model and studied the influence of absorption in the top and bottom layers on the measured reflectivity. Morgan et al. ${ }^{17,18}$ analyzed the spatial distributions of the scattered polarized light using a two-layer isotropic skin model with absorption. In our previous works for studying the penetration depth of polarization imaging, we have developed an anisotropic skin scattering model with isotropic top layer and anisotropic bottom layer. ${ }^{13}$ This model did not include absorption.

In this paper, we study the melanoma using the RLPI method, and compare the imaging contrasts with those of DPI and DOPI methods. We also propose an absorption-scattering model with an isotropic top layer and an anisotropic bottom layer based on our previous works. Monte Carlo (MC) simulations based on this model are carried on to study the influence of scattering and absorption on the contrast of the polarization imaging.

\section{Material and Method}

\subsection{Experimental setup and materials}

The RLPI system used in this paper is same as our previous work. ${ }^{12}$ The schematic of experimental setup is shown in Fig. 1. Light from a 1 W LED (650 $\mathrm{nm}$ ) is collimated by a lens (L1, focal length $50 \mathrm{~mm}$, Daheng Optics, China), polarized by a linear polarizer (P1, extinction ratio 500:1) and irradiates obliquely on the sample. The backscattered light from the sample passes through another polarizer (P2, extinction ratio 500:1). Then the scattered photons are collected by another lens (L2, focal length $50 \mathrm{~mm}$, Daheng Optics, China) and recorded by a CCD camera (Q-imaging RETIGA EXi). To avoid surface glare, a glass plate is placed in close contact to the sample. During RLPI imaging, both the incident polarization angle $\left(\theta_{i}\right)$ and detection polarization angle $\left(\theta_{s}\right)$ are varied by rotating polarizers $\mathrm{P} 1$ and $\mathrm{P} 2$.

The samples in this study are from male nude mice (Guangdong Medical Animal Experimental Center, Guangzhou, China). Tumor samples on the abdominal skin of nude mice are obtained by injection of human melanoma (B16) cells, which is a commonly used skin carcinoma model for animals. In the experiments, the carcinoma areas on the skin tissues are determined by histological examinations.

\subsection{Polarization imaging methods}

In RLPI measurements, we record a series of intensity images corresponding to different incident and detection polarization states, $I\left(\theta_{i}, \theta_{s}\right)$ and $I$

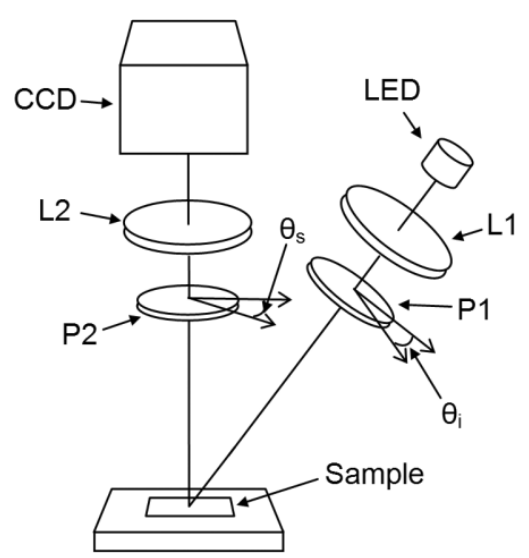

Fig. 1. Schematic of the RLPI system, P: polarizer, L: lens. P1 and P2 can be rotated around their optical axes to vary the polarization angles for both incidence $\left(\theta_{i}\right)$ and detection $\left(\theta_{s}\right)$. 
$\left(\theta_{i}, \theta_{s}+\pi / 2\right)$, and then calculate the linear differential polarization (LDP, Eq. (1)) at each pixel:

$$
\operatorname{LDP}\left(\theta_{i}, \theta_{s}\right)=I\left(\theta_{i}, \theta_{s}\right)-I\left(\theta_{i}, \theta_{s}+\pi / 2\right) .
$$

The LDP images can be fitted to an analytical expression (Eq. (2)) ${ }^{11}$ :

$$
\begin{gathered}
\operatorname{LDP}\left(\theta_{i}, \theta_{s}\right)=\frac{1}{2} I_{i} \sqrt{A \cos \left(4 \theta_{i}-\varphi_{1}\right)} \\
+B \cos \left[2 \theta_{i}-\varphi_{2}\left(\theta_{s}\right)\right] \\
+\frac{1}{2} I_{i} C \cos \left(2 \theta_{s}-\varphi_{3}\right) .
\end{gathered}
$$

From Eq. (2), we can obtain a set of new parameters $A, B, C, \varphi_{1}$ and $\varphi_{3}$. These parameters are independent of incident polarization angle $\left(\theta_{i}\right)$ and detection polarization angle $\left(\theta_{s}\right)$. More importantly, these parameters are related to different structural or optical properties of the sample, such as density, shape and size of the scatterers, as well as the direction and alignment of the fibers. ${ }^{11-13}$ The RLPI parameters can be expressed as functions of Mueller matrix elements. We also defined $G=A / B$ and proved it can quantitatively characterize the anisotropic properties of the sample. ${ }^{11}$

Moreover, we realize DPI and DOPI in the same RLPI measurement. Difference of polarization (DP) and degree of polarization (DOP) are obtained corresponding to specific incident and detection polarization angles, $\theta_{s}=\theta_{i}$ and $\theta_{s}=\theta_{i}+\pi / 2$ shown as Eqs. (3) and (4).

$$
\begin{gathered}
D P=I_{\|}-I_{\perp}=I\left(\theta_{i}, \theta_{i}\right)-I\left(\theta_{i}, \theta_{i}+\pi / 2\right), \\
D O P=\frac{I_{\|}-I_{\perp}}{I_{\|}+I_{\perp}}=\frac{I\left(\theta_{i}, \theta_{i}\right)-I\left(\theta_{i}, \theta_{i}+\pi / 2\right)}{I\left(\theta_{i}, \theta_{i}\right)+I\left(\theta_{i}, \theta_{i}+\pi / 2\right)} .
\end{gathered}
$$

\subsection{MC simulation}

For a better understanding of the experimental results, we use a MC program to simulate the propagation and scattering of polarized photon in the tissue samples. The MC simulation program is based on a sphere-cylinder scattering model (SCSM) ${ }^{19}$ which contains spheres and infinitely long cylinders to mimic the isotropic and anisotropic scatterers in tissues.

The detailed process of MC simulations can be found in our previous paper. ${ }^{20}$ For comparisons between the simulated and experimental results, parameters of the simulations are set according to published tissue data. The refractive indices of the scatterers and surrounding medium are 1.4 and 1.33, respectively. ${ }^{21}$ The diameters and scattering coefficients of the spherical and cylindrical scatterers are set according to Refs. 21-25. For the study of polarization imaging of melanoma samples, absorption is added in the MC simulations as a variable of the surrounding medium.

\section{Results and Discussion}

\subsection{Polarization imaging of melanoma}

The experimental system in Fig. 1 can take RLPI, DPI, DOPI and ordinary intensity imaging in the same measurement. We use this system on two melanoma samples, the results are shown in Figs. 2 and 3 , respectively.

Figures 2(a) to 2(h) show the experimental results of intensity, DP, DOP and the five RLPI parameters $\left(G, A, B, C, \varphi_{3} / 2\right)$ for melanoma sample 1 . The area marked with a yellow circle in Fig. 2 is the cancerous tissue. We can conclude from Figs. 2(d) to 2(h) that among the five RLPI parameters, $A$ and $G$ provide the best contrasts between the normal and the cancerous tissues. Besides, compared with parameter $A$, parameter $G$ shows more fibrous texture, corresponding to structural information of the sample.

To compare the contrasts of the four imaging parameters: intensity in Fig. 2(a), DP for DPI in Fig. 2(b), DOP for DOPI in Fig. 2(c) and $G$ for RLPI in Fig. 2(d), we use two squares to mark the normal and cancerous tissues, respectively, then calculate the contrast of each parameter based on the mean values within the two squares, as shown in the inserts at the bottom left corner of each picture. It can be observed from Figs. 2(a) to 2(d) that, the contrast for intensity imaging is 0.26 ; DPI and DOPI result in lower contrasts (0.06 and 0.21), which are possibly due to the absorption of the sample. For the same reason, the RLPI parameter $G$ has lower contrast (0.19) than that of DOPI but provides more structural information of the sample compared with DPI and DOPI methods.

Figures 3(a) to $3(\mathrm{~h})$ show the experimental results of intensity, DPI, DOPI and the five RLPI parameters $\left(G, A, B, C, \varphi_{3} / 2\right)$ for melanoma sample 2. We can observe from Fig. 3(a) that, the melanoma in sample 2 cannot be distinguished 


\section{Li et al.}

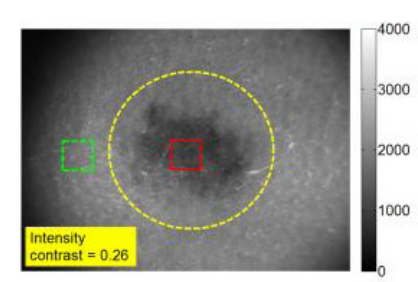

(a)

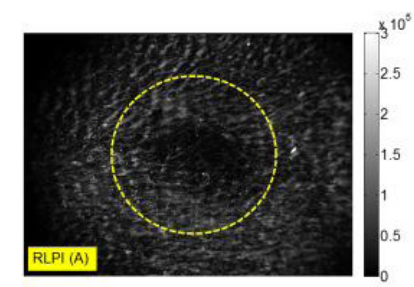

(e)

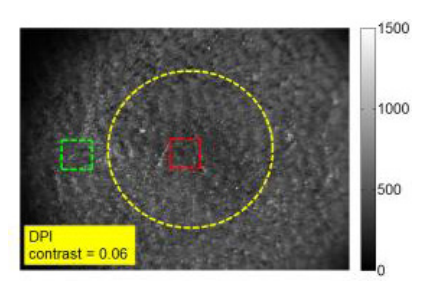

(b)

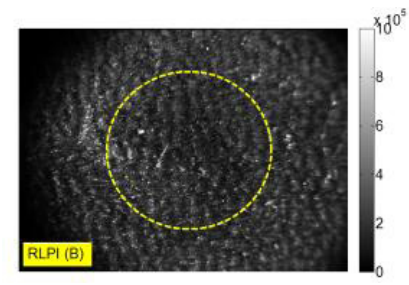

(f)

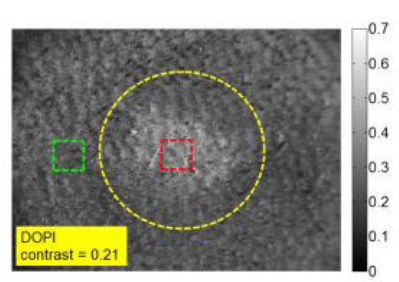

(c)

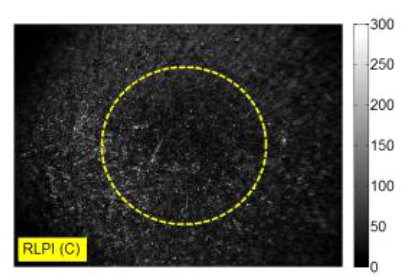

(g)

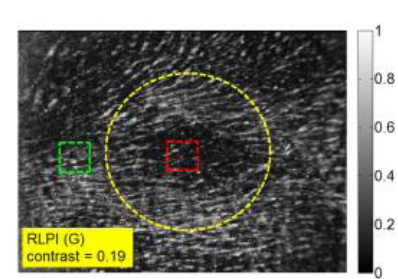

(d)

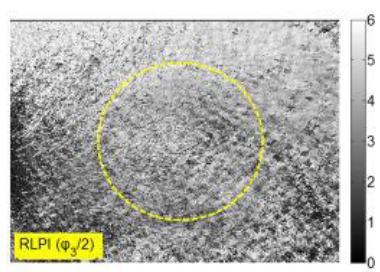

(h)

Fig. 2. Experimental results of sample 1: (a) intensity, (b) DP, (c) DOP, (d) $G$, (e) $A$, (f) $B$, (g) $C$ and (h) $\varphi_{3} / 2$, for a nude mouse's melanoma with carcinoma B16. The carcinoma areas are marked by yellow circles. Contrast between the healthy (marked as green squares) and cancerous skin tissues (marked as red squares) for intensity, DP, DOP and $G$ are: 0.26, 0.06, 0.21 and 0.19.

clearly from intensity image, which is different from sample 1. However, it can be concluded from Figs. 3(b) to 3(d) that the polarization imaging parameters can be used to detect the cancerous tissues. Especially, we can obtain more detailed information from the RLPI parameter $G$ image (Fig. 3(d)). The difference between sample 1 and sample 2 may due to the depth of the cancerous tissues. Thus we can say that the RLPI method can demarcate cancer margins better than DPI and DOPI, when the cancerous area lies in a certain depth below the epidermis.

As shown in Figs. 2 and 3, various factors such as scattering, absorption and location depth may affect the imaging results. In order to analyze the effects of these factors separately, we establish a

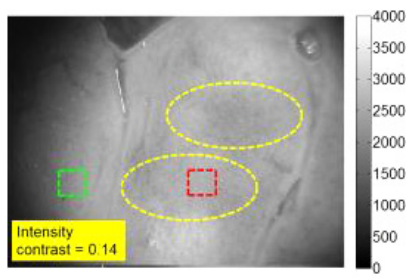

(a)

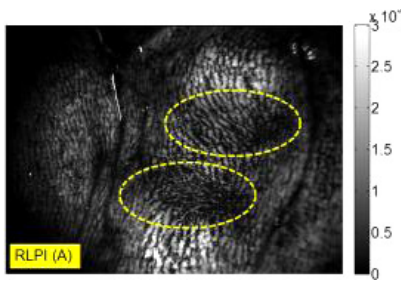

(e)

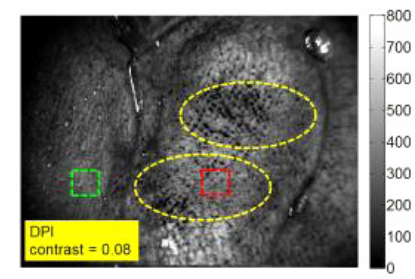

(b)

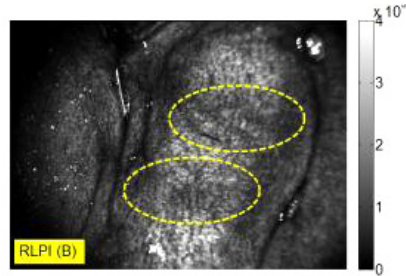

(f)

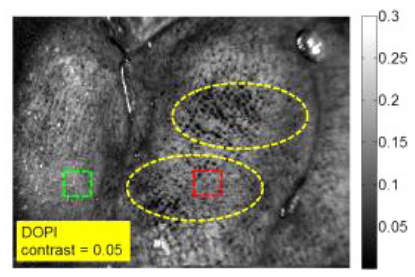

(c)

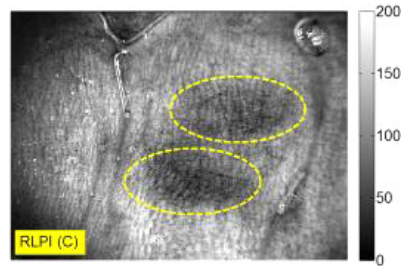

(g)

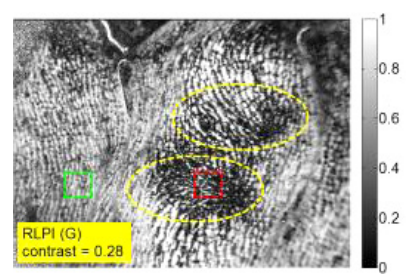

(d)

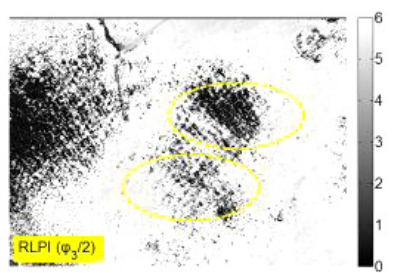

(h)

Fig. 3. Experimental results of sample 2: (a) intensity, (b) DP, (c) DOP, (d) $G$, (e) $A$, (f) $B$, (g) $\varphi_{3} / 2$ and (h) $G$, for a nude mouse's melanoma with carcinoma B16. The carcinoma areas are marked by yellow circle frames. Contrast between the healthy (marked as green squares) and cancerous skin tissues (marked as red squares) for intensity, DP, DOP and $G$ are: 0.14, 0.08, 0.05 and 0.28. 


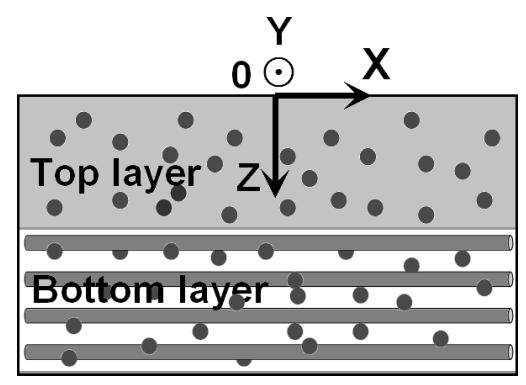

Fig. 4. Schematic diagram of two-layer model for cancerous skin tissues. The top layer is isotropic, the bottom layer is anisotropic and both layers may include absorption effect.

model for skin and compare the experimental results with Monte Carlo simulation based on this model.

\subsection{Model and simulation results}

\subsubsection{Simulation model for cancerous skin tissues}

Based on the pathological characteristics of melanoma ${ }^{26}$ and our previous work, ${ }^{13}$ we propose a twolayer model for the cancerous skin tissues as shown in Fig. 4. The skin model has a top isotropic layer (simulated by spherical scatterers) and a bottom anisotropic layer (simulated by spherical and cylindrical scatterers). Both layers may include absorption effect. For the anisotropic lesion with absorption, two major factors are considered: the

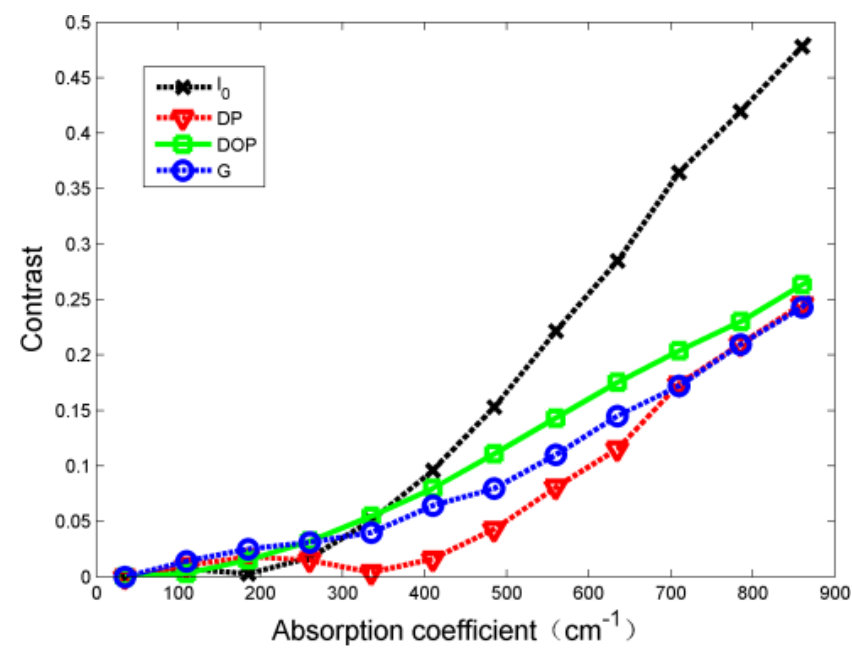

(a) absorption coefficient corresponding to the melanin proliferation, and the scattering coefficient, which should be strongly correlated to cell proliferation.

\subsubsection{The effect of absorption coefficient on polarization imaging for melanoma}

First, we study the effect of absorption coefficient on the polarization imaging. To simulate the experimental samples, the parameters in MC simulations are set accordingly: The top isotropic layer with thickness of $20 \mu \mathrm{m}$ consists of spherical scatterers with diameter of $0.46 \mu \mathrm{m}$ and refractive index of 1.4. ${ }^{22,23}$ The bottom layer with infinite thickness consists of the same spherical scatterers and the cylindrical scatterers with diameter of $1 \mu \mathrm{m}$ and refractive index of $1.4 .^{24}$ The mouse and human skins are of similar structures and properties. ${ }^{25}$ The average absorption coefficients of the normal skin tissues are $35 \mathrm{~cm}^{-1}$ for epidermis (top layer) and $2.7 \mathrm{~cm}^{-1}$ for dermis (bottom layer) as reported in Ref. 23. To simulate the cancer-induced melanin proliferation, during the simulation we change the absorption coefficients from $35 \mathrm{~cm}^{-1}$ for top layer and $2.7 \mathrm{~cm}^{-1}$ for bottom layers to $900 \mathrm{~cm}^{-1}$ and $250 \mathrm{~cm}^{-1}$, respectively. ${ }^{21}$ Polarization imaging parameters of intensity, DP, DOP and RLPI parameter $G$ are calculated and the relative contrasts between the cancerous and the normal tissue are obtained, as shown in Fig. 5.

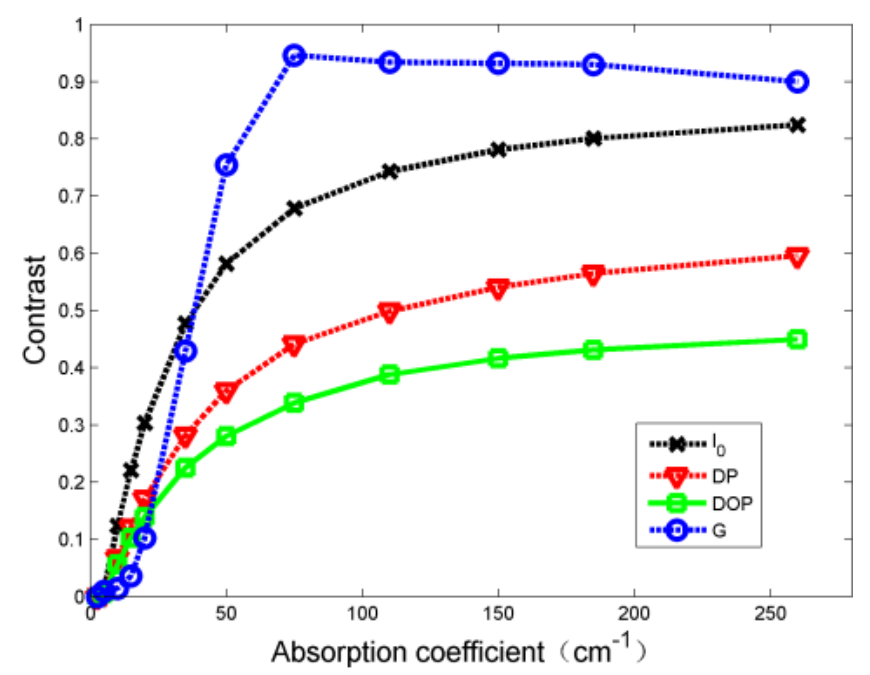

(b)

Fig. 5. Simulated relative contrasts of four imaging parameters: intensity, DP, DOP, RLPI parameter $G$ at different absorption coefficients for a two-layer structure with top isotropic layer and bottom anisotropic layer. (a) The absorption coefficient varies from 35 to $900 \mathrm{~cm}^{-1}$ in top layer, (b) The absorption coefficient varies from 2.7 to $250 \mathrm{~cm}^{-1}$ in bottom layer. 


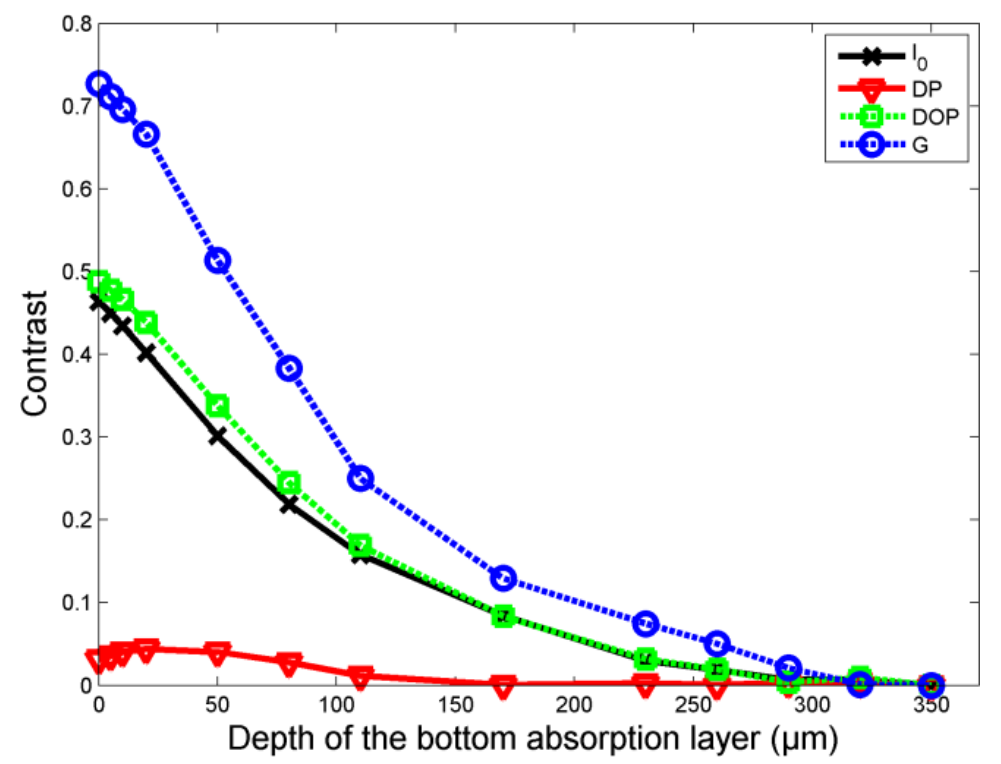

Fig. 6. Monte Carlo simulated relative contrasts of four imaging parameters: intensity, DP, DOP, RLPI parameter $G$, at different depths of the bottom absorption layer for a two-layer structure with top isotropic layer and bottom anisotropic layer. The absorption coefficient of the bottom layer is $20 \mathrm{~cm}^{-1}$. The depth of the bottom layer varies from 0 to $350 \mu \mathrm{m}$.

We can conclude from Figs. 5(a) and 5(b) that the relative contrasts of all the four parameters rise with the increase of absorption coefficient for the top and the bottom layer. Furthermore, the orders of the four contrasts in Figs. 5(a) and 5 (b) match with the experimental results shown in Fig. 2 (intensity $>\mathrm{DOP}>G>\mathrm{DP}$ ) and Fig. 3 $(G>$ intensity $>\mathrm{DP}>\mathrm{DOP})$, respectively. In addition, it can be observed from Figs. 5(a) and 5(b) that, the four parameters change more dramatically with the increase of absorption in the bottom layer than the top layer. This is because that the multiple scattered photons act differently on the thin top layer and the infinite bottom layer when absorption exists.

\subsubsection{The depth effect of the bottom absorption layer}

As mentioned in Sec. 3.2.2, the absorption of the bottom layer affects the polarization imaging more dramatically than that of the top layer. Furthermore, nodular malignant melanoma has no invasion to the epidermis (top layer), whose thickness varies in different areas of human body. So we further study the effect of depth for the bottom absorption layer on the polarization imaging.

We study the depth effect of the bottom absorption layer on the polarization imaging of melanoma. The parameters in MC simulations are the same as Sec. 3.2.2. The depth of the bottom absorption layer varies from 0 to $350 \mu \mathrm{m}$ and the absorption coefficient is $20 \mathrm{~cm}^{-1}$. For calculating the contrast, we use the condition when the bottom layer has infinite depth as the reference value, the results are shown in Fig. 6. We can see from Fig. 6 that, as bottom absorption layer moves deeper, the absorption effect reduces and the anisotropy decreases. It also can be concluded from Fig. 6 that, $G$ is the most sensitive parameter to anisotropy, which agrees well with the absorption-free results in our previous works. ${ }^{13}$

\subsubsection{The effect of scattering coefficient on the polarization imaging for melanoma}

Furthermore, we study the effect of scattering coefficient on the relative contrasts of different polarization imaging techniques. The parameters in MC simulations are the same as in Sec. 3.2.2. The scattering coefficients are $450 \mathrm{~cm}^{-1}$ for epidermis (the top layer) and $187.5 \mathrm{~cm}^{-1}$ for dermis (the bottom layer), and changed to $1000 \mathrm{~cm}^{-1}$ for both layers to simulate the cancer-induced cell proliferation. ${ }^{21,23}$ Polarization imaging parameters of intensity, DP, DOP and RLPI parameter $G$ are calculated and the contrasts between the cancerous and the normal tissue are obtained, as shown in Fig. 7.

It can be observed in Figs. 7(a) and 7(b) that the relative contrasts of all the four parameters rise 


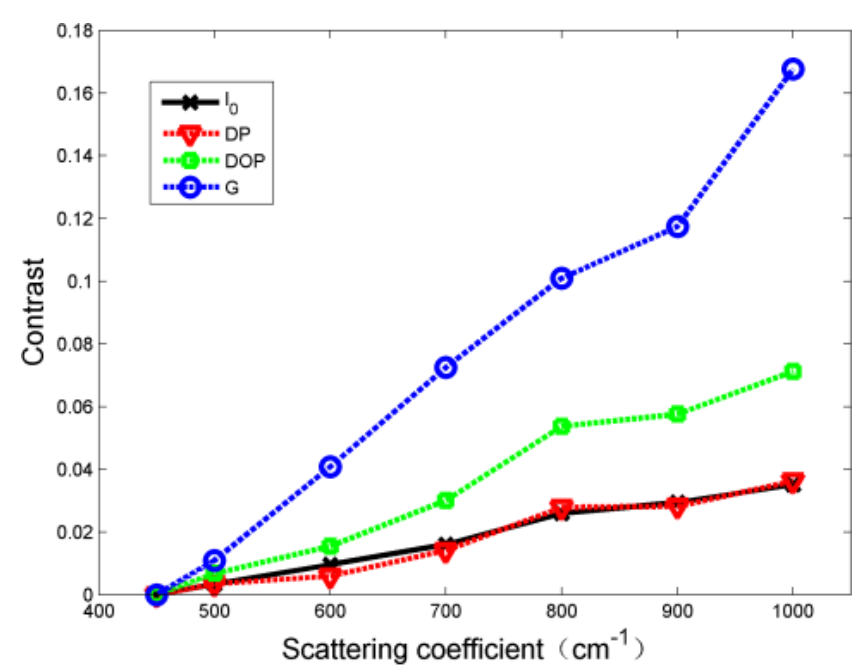

(a)

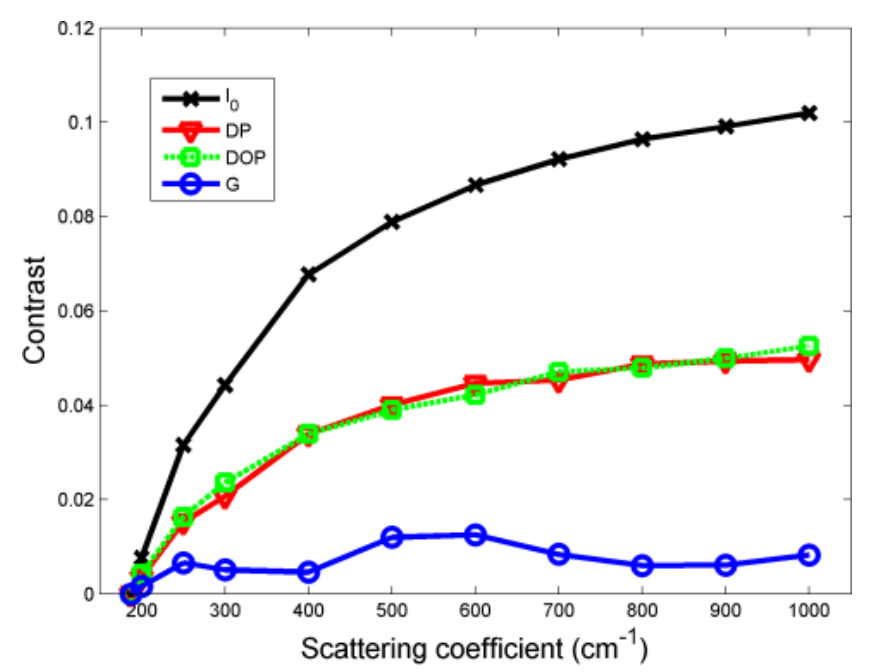

(b)

Fig. 7. Monte Carlo simulated relative contrasts of four imaging parameters: intensity, DP, DOP, RLPI parameter $G$ at different scattering coefficients for a two-layer structure with top isotropic layer and bottom anisotropic layer. (a) The scattering coefficient varies from 450 to $1000 \mathrm{~cm}^{-1}$ in top layer, (b) The scattering coefficient varies from 187.5 to $1000 \mathrm{~cm}^{-1}$ in bottom layer.

with the increase of scattering coefficient for the top and the bottom layer, respectively. However, the orders of the four contrasts in Figs. 7(a) and 7(b) are different. The sequence of four contrasts in descending order is $G$, DOP, intensity, DP when the scattering coefficient of the top layer changes (Fig. 7 (a)). The sequence is intensity, DP, DOP, $G$ when the scattering coefficient of the bottom layer changes (Fig. 7(b)). Moreover, these sequences are different from the experimental results shown in Fig. 2 (intensity, DOP, G, DP) and Fig. 3 (G, intensity, DP, DOP). During the simulation, we also adjust the structural parameters including sizes (diameters of the spherical and cylindrical scatterers) and scattering coefficients. We find that the two-layer model with only scattering cannot generate results matching the experiments of melanoma.

In short, comparing the simulation results in Figs. 5 and 7 with experimental results in Figs. 2 and 3 , we can conclude that the scattering model with absorption effect is suitable for the study of polarization imaging of melanoma.

\section{Conclusion}

In this paper, we apply the RLPI method to the study of melanoma and compare the RLPI parameters with DPI and DOPI methods. Relative contrasts of these polarization imaging methods are measured experimentally. For better understanding of the contrast mechanism in the polarization imaging of melanoma, we develop the Monte Carlo simulations based on a two-layer absorption-scattering model with an isotropic layer at the top and an anisotropic layer at the bottom. By comparing the simulation results with the experimental results, we find that the two-layer model can reproduce the relative contrast for polarization images observed in experiments. The simulation results also show that, the parameters of polarization imaging change more dramatically with the variation of absorption in the bottom layer than the top layer. Furthermore, we also have some discussions on the impact of the depth of absorption layer on polarization imaging. The absorption-scattering model for skins provides a useful tool to analyze the contrast mechanism of polarization imaging of melanoma.

\section{Acknowledgments}

This work was supported by the National Natural Science Foundation of China (NSFC) Grant Nos. 10974114, 11174178, 61205199, and the Knowledge Innovation Program of Basic Research Projects of Shenzhen Grant No. JCY20130402145002404.

\section{References}

1. V. V. Tuchin, L. V. Wang, D. A. Zimnyakov, "Optical polarization in biomedical applications," Tissue structure and optical models, E. Greenbaum, 
Ed., Springer, Berlin Heidelberg New York (2006), pp. 7-28.

2. S. K. Gayen, R. R. Alfano. "Emerging optical biomedical imaging techniques," Opt. Photonics News 7(3), 17-22 (1996).

3. V. Backman, M. B. Wallace, L. T. Perelman, J. T. Arendt, R. Gurjar, M. G. Muller, Q. Zhang, G. Zonios, E. Kline, T. McGillican, S. Shapshay, T. Valdez, K. Badizadegan, J. M. Crawford, M. Fitzmaurice, S. Kabani, H. S. Levin, M. Seiler, R. R. Dasari, I. Itzkan, J. Van Dam, M. S. Feld, "Detection of preinvasive cancer cells," Nature 406 (6791), 35-36 (2000).

4. A. Pierangelo, A. Nazac, A. Benali, P. Validire, H. Cohen, T. Novikova, B. Haj Ibrahim, S. Manhas, C. Fallet, M. Antonelli, A. Martino, "Polarimetric imaging of uterine cervix: A case study," Opt. Exp. 21(12), 14120-14130 (2013).

5. R. S. Gurjar, V. Backman, L. T. Perelman, I. Georgakoudi, K. Badizadegan, I. Itzkan, R. R. Dasari, M. S. Feld, "Imaging human epithelial properties with polarized light-scattering spectroscopy," Nat. Med. 7(11), 1245-1248 (2001).

6. Y. A. Ushenko, O. I. Telenga, A. P. Peresunko, O. K. Numan, "New parameter for describing and analyzing the optical-anisotropic properties of biological tissues," J. Innov. Opt. Health Sci. 4(4), 463475 (2011).

7. S. L. Jacques, J. C. Ramella-Roman, K. Lee, "Imaging skin pathology with polarized light," J. Biomed. Opt. 7(3), 329-340 (2002).

8. J. C. Ramella-Roman, K. Lee, S. A. Parahl, S. L. Jacques, "Design, testing, and clinical studies of a handheld polarized light camera," J. Biomed. Opt. 9(6), 1305-1310 (2004).

9. S. L. Jacques, R. Samathama, S. Isenhath, K. Lee, "Polarized light camera to guide surgical excision of skin cancers," Proc. SPIE, 6842, 684201 (2008).

10. R. R. Anderson, "Polarized light examination and photography of the skin," Arch. Dermatol. 127(7), 1000-1005 (1991).

11. R. Liao, N. Zeng, X. Y. Jiang, D. Z. Li, T. L. Yun, Y. H. He, H. Ma, "Rotating linear polarization imaging technique for anisotropic tissues," $J$. Biomed. Opt. 15(3), 036014 (2010).

12. D. Z. Li, H. H. He, N. Zeng, E Du, R. Liao, Y. H. He, H. Ma, S. X. Liu, M. H. Li, "Polarization imaging and scattering model of cancerous liver tissues," J. Innov. Opt. Health Sci. 6(3), 1350025 (2013).

13. R. Liao, N. Zeng, D. Z. Li, T. L. Yun, Y. H. He, H. Ma, "Penetration depth of linear polarization imaging for two-layer anisotropic samples," Appl. Opt. 15(23), 4681-4687 (2011).

14. L. Li, C. S. L. Ng, "Rendering human skin using a multilayer reflection model," Int. J. Comput. Math. 3(1), 44-53 (2009).

15. G. Mantis, G. Zonios, "Simple two-layer reflectance model for biological tissue applications," Appl. Opt. 48(18), 3490-3496 (2009).

16. G. Zonios, A. Dimou, "Simple two-layer reflectance model for biological tissue applications: Lower absorbing layer," Appl. Opt. 49(27), 5026-5031 (2010).

17. S. P. Morgan, M. E. Ridgway, "Polarization properties of light backscattered from a two layer scattering medium," Opt. Exp. 7(12), 395-402 (2000).

18. I. M. Stockford, S. P. Morgan, P. C. Y. Chang, J. G. Walker, "Analysis of the spatial distribution of polarized light backscattered from layered scattering media," J. Biomed. Opt. 7(3), 313-320 (2002).

19. H. H. He, N. Zeng, R. Liao, T. L. Yun, W. Li, Y. H. He, H. Ma, "Application of sphere-cylinder scattering model to skeletal muscle," Opt. Exp. 18(14), 15104-15112 (2010).

20. T. L. Yun, N. Zeng, W. Li, D. Z. Li, X. Y. Jiang, H. Ma, "Monte Carlo simulation of polarized photon scattering in anisotropic media," Opt. Exp. 17(19), 16591-16602 (2009).

21. V. V. Tuchin, Tissue Optics: Light Scattering Methods and Instruments for Medical Diagnosis, SPIE Press, Bellingham (2007).

22. K. Calabro, A. Curtis, J. R. Galarneau, T. Krucker, I. J. Bigio, "Gender variations in the optical properties of skin in murine animal models," J. Biomed. Opt. 16(1), 011008 (2011).

23. M. J. C. van Gemert, S. L. Jacques, H. J. C. M. Sterenborg, W. M. Star, "Skin optics," IEEE Trans. Biomed. Eng. 36(12), 1146-1154 (1989).

24. M. Raspanti, M. Viola, M. Sonaggere, M. E. Tira, R. Tenni. "Collagen fibril structure is affected by collagen concentration and decorin," Biomacromolecules 8(7), 2087-2091 (2007).

25. D. D. Kim, Y. W. Chien, "Transdermal delivery of dideoxynucleoside - type anti IV HIV drugs. 2. The effect of vehicle and enhancer on skin permeation," J. Pharm. Sci. 85(2), 214-219 (1996).

26. U. Leiter, F. Meier, B. Schittek, C. Garbe, "The natural course of cutaneous melanoma," J. Surg. Oncol. 86(4), 172-178 (2004). 